Detection of Hohlraum Target Position for Laser Fusion Experiments

A. A. S. Awwal

July 16, 2010

SPIE Optics and Photonics 2010

San Diego, CA, United States

August 1, 2010 through August 5, 2010 
This document was prepared as an account of work sponsored by an agency of the United States government. Neither the United States government nor Lawrence Livermore National Security, LLC, nor any of their employees makes any warranty, expressed or implied, or assumes any legal liability or responsibility for the accuracy, completeness, or usefulness of any information, apparatus, product, or process disclosed, or represents that its use would not infringe privately owned rights. Reference herein to any specific commercial product, process, or service by trade name, trademark, manufacturer, or otherwise does not necessarily constitute or imply its endorsement, recommendation, or favoring by the United States government or Lawrence Livermore National Security, LLC. The views and opinions of authors expressed herein do not necessarily state or reflect those of the United States government or Lawrence Livermore National Security, LLC, and shall not be used for advertising or product endorsement purposes. 


\title{
Detection of Hohlraum Target Position for Laser Fusion Experiments
}

\author{
Abdul A. S. Awwal \\ Integrated Computer Control System, National Ignition Facility \\ Laser Science Engineering and Operations, \\ Lawrence Livermore National Laboratory, Livermore, CA. 94551 \\ E-mail: awwall@1lnl.gov
}

\begin{abstract}
A hohlraum is a cylindrical structure that holds a laser fusion target at the National Ignition Facility. It must be aligned properly for all the 192 laser beams to hit the target and cause a fusion reaction. Video images of the hohlraum are used to align the hohlraum to the required position. A matched filtering based approach is used to locate the circular alignment fiducial of the hohlraum. One of the challenges of the automatic alignment algorithm is the presence of a number of nearly concentric features from which only one will provide the valid position information. The problem is compounded by blurring of relevant features by defocus or insufficient illumination and amplification of non-relevant features. It is shown that to identify the appropriate fiducial; the shape (or size) in addition to amplitude of correlation peak must be considered.
\end{abstract}

Key word: Pattern recognition, image processing, correlation shape, laser alignment

\section{INTRODUCTION}

The National Ignition Facility (NIF), developed at the Lawrence Livermore National Laboratory, is a stadium-sized facility that contains a 192-beam, 1.8-megajoule, 500-terawatt, ultraviolet laser system dedicated to the study of inertial confinement fusion and the physics of matter at extreme energy densities and pressures [1]. Careful alignment of this high energy pulsed laser to the hohlraum target is crucial [2,3] for producing high-energy density and pressure conditions leading to a controlled fusion reaction. An automatic alignment (AA) system was designed and implemented to ensure successful delivery of high energy pulse in each of the 192 laser beams [4].

The integrated automated control system performs the alignment of the laser beams by measuring the current position of the beam, and controlling mirrors and other devices, and adjusting the beam to a desired reference location. The position of the beam is determined by algorithms processing beam images with or without spatial fiducials. Numerous types of images are being processed throughout the beam path of each laser beam as shown in Fig. 1. In the current work, instead of aligning a bright laser beam, an object is being aligned, which may lack proper illumination and imaging condition, affecting the quality of the image.

Matched filtering has been successfully utilized to determine the location of beam fiducials with distinct shapes [5-8]. The matched filtering allows one to find beam position without noise filtering, and is tolerant to local intensity variation. When features are matched, higher spatio-temporal stability of beam position is achieved. Additionally the algorithm can accommodate various types and sizes of the fiducials.

Matched filtering techniques rely on the fact that the position of the highest correlation peak is an indicator of the position of the fiducial. However, when the feature strength is weak compared to other features, considered noise, this assumption of maximum correlation peak appearing at the fiducial position becomes problematic. The highest peaks tend to favor detection of false objects with better signal level. The hohlraum image is an example of such a challenging 
situation. In these images, the fiducials of interest are sometimes very well defined and sometimes out of focus by design, resulting in low contrast of the image feature. In this work, we demonstrate how intelligent processing of the correlation plane enables one to identify the object of interest in the presence of competing bright objects of noninterest.
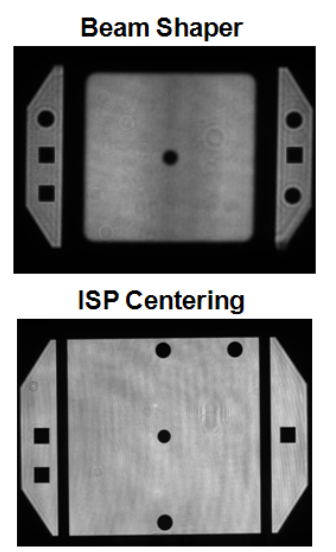

Darkstop

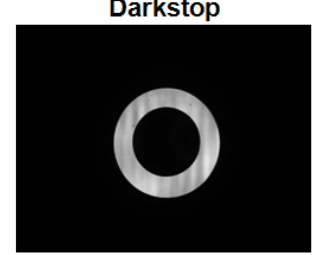

Csf_p3_pinhole

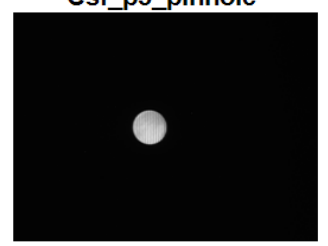

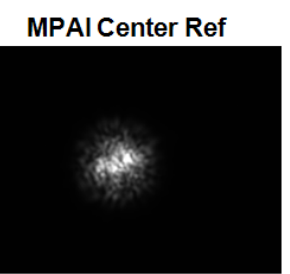

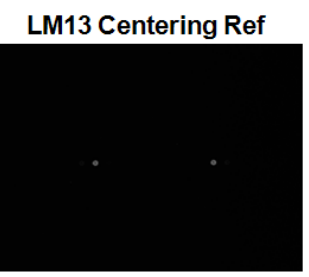

FOA Centering Beam

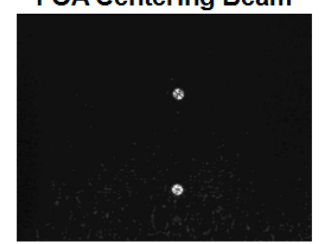

KDP w phase plate

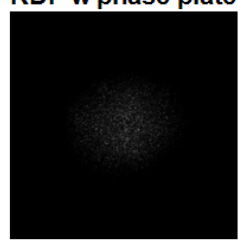

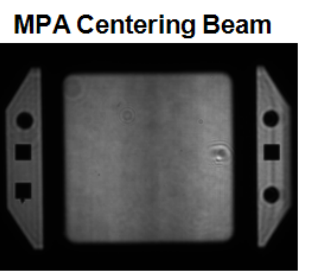

LM3 Center Beam

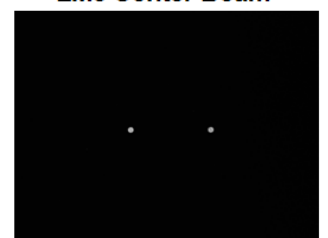

KDP Back Reflection

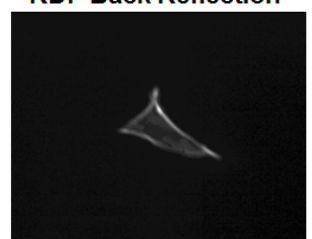

TAS Position

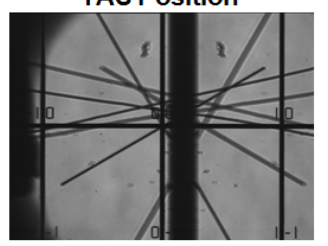

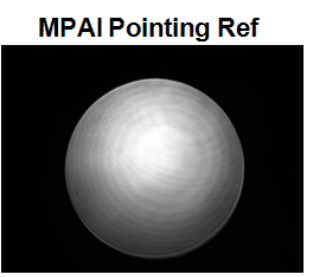

ISP_LM3 beam

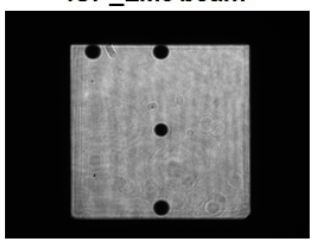

Target Chamber Focus

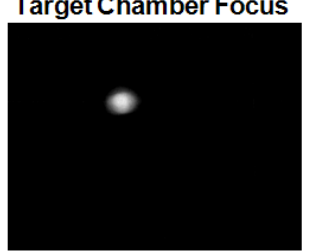

Pin Hole Check

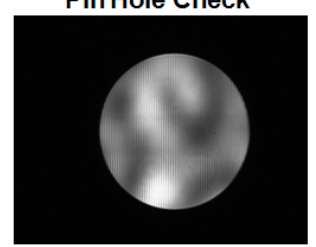

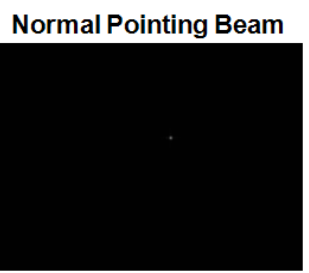

LOIS

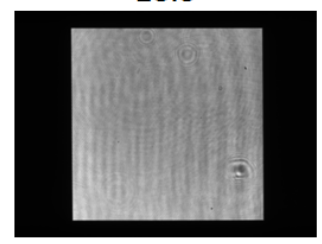

TAS Tip/Tilt

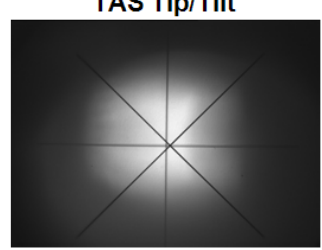

Tsf_p1_beam

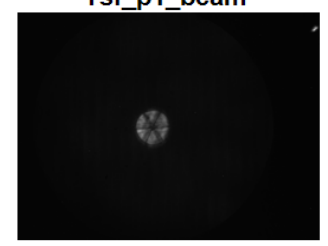

Fig. 1: Varieties of image processed by automatic alignment algorithms. The names refer to different positions or optics in the NIF beam lines.

\section{BASICS OF MATCHED FILTERING}

The algorithm utilized here to detect the object of interest is based on classical matched filter (CMF). The computational advantage of fast Fourier transform motivates us to opt for the Fourier domain description of the CMF. Assuming that the Fourier transform of the object function $f(\mathrm{x}, \mathrm{y})$ be denoted by

$$
F\left(U_{x}, U_{y}\right)=\left|F\left(U_{x}, U_{y}\right)\right| \exp \left(j \Phi\left(U_{x}, U_{y}\right)\right)
$$

the CMF $[9,10]$ corresponding to this function $f(x, y)$, is given by the complex conjugate of the input Fourier spectrum denoted by Eq. 2,

$$
H_{C M F}\left(U_{x}, U_{y}\right)=F^{*}\left(U_{x}, U_{y}\right)=\left|F\left(U_{x}, U_{y}\right)\right| \exp \left(-j \Phi\left(U_{x}, U_{y}\right)\right)
$$


The product of Eqs. (1) and (2) produces the correlation in the Fourier domain, then the inverse Fourier transform produces the autocorrelation. The performance of the matched filter can be further enhanced by extracting the edge of the image and using the edge of the to-be-detected features as the filter. This has an equivalent effect of high pass filtering the correlation output, thus increasing the sharpness of the peaks [11,12].

The position of the object can be found from the position of the cross correlation peak, autocorrelation peak, and the position of the template using the Eqs. 3-4

$$
\begin{aligned}
& \mathrm{x}_{\text {pos }}=\mathrm{x}_{\text {cross }}-\mathrm{x}_{\text {auto }}+\mathrm{x}_{\mathrm{c}} \\
& \mathrm{y}_{\text {pos }}=\mathrm{y}_{\text {cross }}-\mathrm{y}_{\text {auto }}+\mathrm{y}_{\mathrm{c}}
\end{aligned}
$$

where $\left(\mathrm{x}_{\mathrm{pos},} \mathrm{y}_{\mathrm{pos}}\right)$ is the to-be-determined position of the pattern in the image plane, ( $\left.\mathrm{x}_{\text {auto, }}, \mathrm{Y}_{\text {auto }}\right)$ is the position of the template autocorrelation peak and $\left(\mathrm{x}_{\text {cross }}, \mathrm{y}_{\text {cross }}\right)$ is the position of the cross correlation peak. The position of the crosscorrelation peak is estimated using a polynomial fit of second order to the correlation peak. The center of the template, $\left(\mathrm{x}_{\mathrm{c}}, \mathrm{y}_{\mathrm{c}}\right)$, and $\left(\mathrm{x}_{\mathrm{auto}}, \mathrm{y}_{\text {auto }}\right)$ is calculated off-line. By centering the template to the center of the image, $\left(\mathrm{x}_{\mathrm{c},}, \mathrm{y}_{\mathrm{c}}\right)$ and $\left(\mathrm{x}_{\text {auto,o }}, \mathrm{Y}_{\text {auto }}\right)$ cancel each other.

\section{ALGORITHM FOR DETECTING FAINT BEAM FIDUCIALS}

The hohlraum target image depicted in Fig. 2 consists of a set of concentric circular features. The designated alignment feature of this object is the circular metallic ring, which contains two notches, as shown clearly in right image of Fig. 2. It is interesting to note that the same feature is faint and fuzzy and barely visible in the left image of Fig. 2, whereas other features are more prominent. Since the right image is well focused, the image feature, i.e., the metallic ring, can be clearly discerned.

\section{Feature with low contrast}

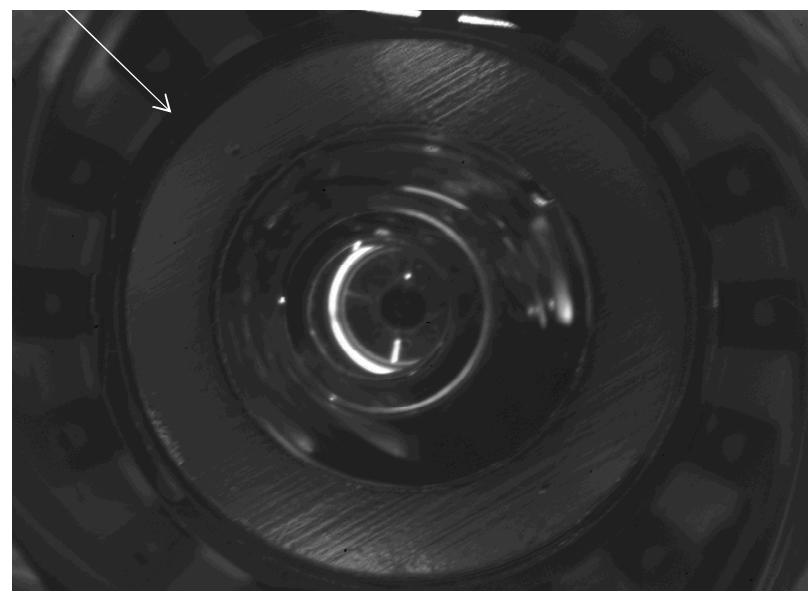

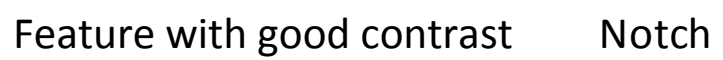

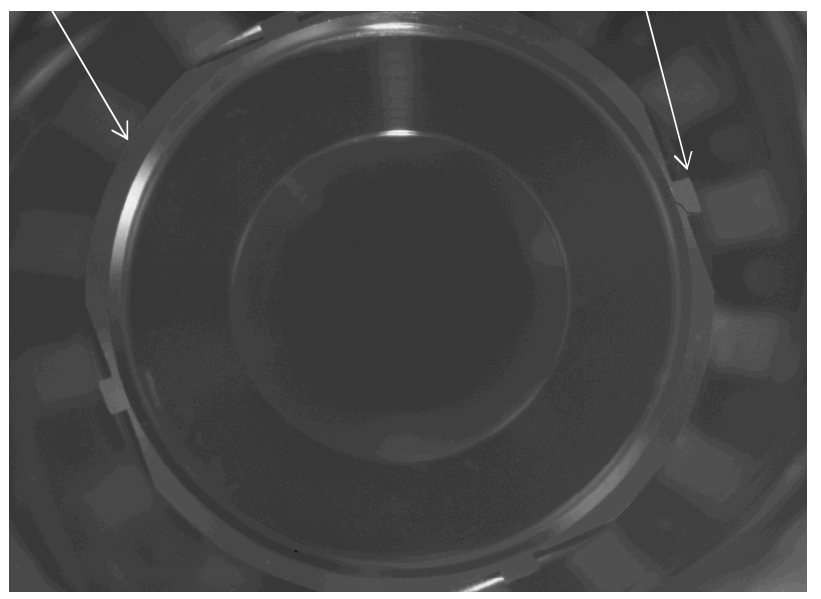

Fig. 2: A view of the hohlraum with showing the fiducial to-be-detected, which is circular edge of hohlraum

The various steps of the detection algorithm are depicted in the block diagram of Fig. 3. In order to detect the metallic ring, a feature extraction (namely edge detection) is performed as a first step. Ideally, we want to detect the ring resulting from this edge detection. Therefore, we choose a template which is a circular ring with a certain thickness and 
radius equal to the nominal radius of the target feature. Since certain amount of variability exists, we vary the radius of the ring and after matched filtering look for the maxima from all the correlation peak values.

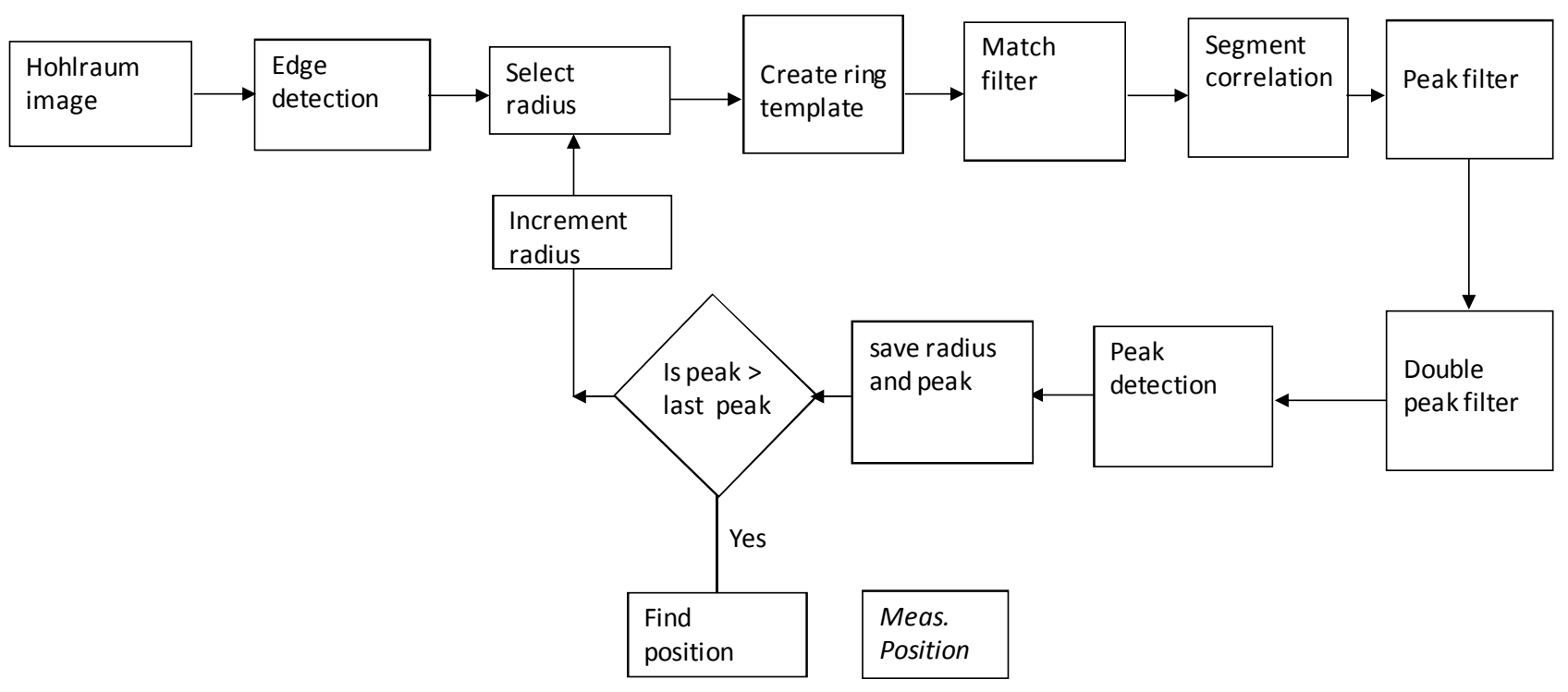

Fig. 3: Block diagram of hohlraum position detection algorithm.

When correlated with the image in Fig. 2, the resulting correlation output is shown in Fig. 4. The output shows a large number of bright peaks corresponding to many false matches. Note also that the correlation between circles of different radii results in a circle, which shows up in correlation output. When there is a match between the input radius and the

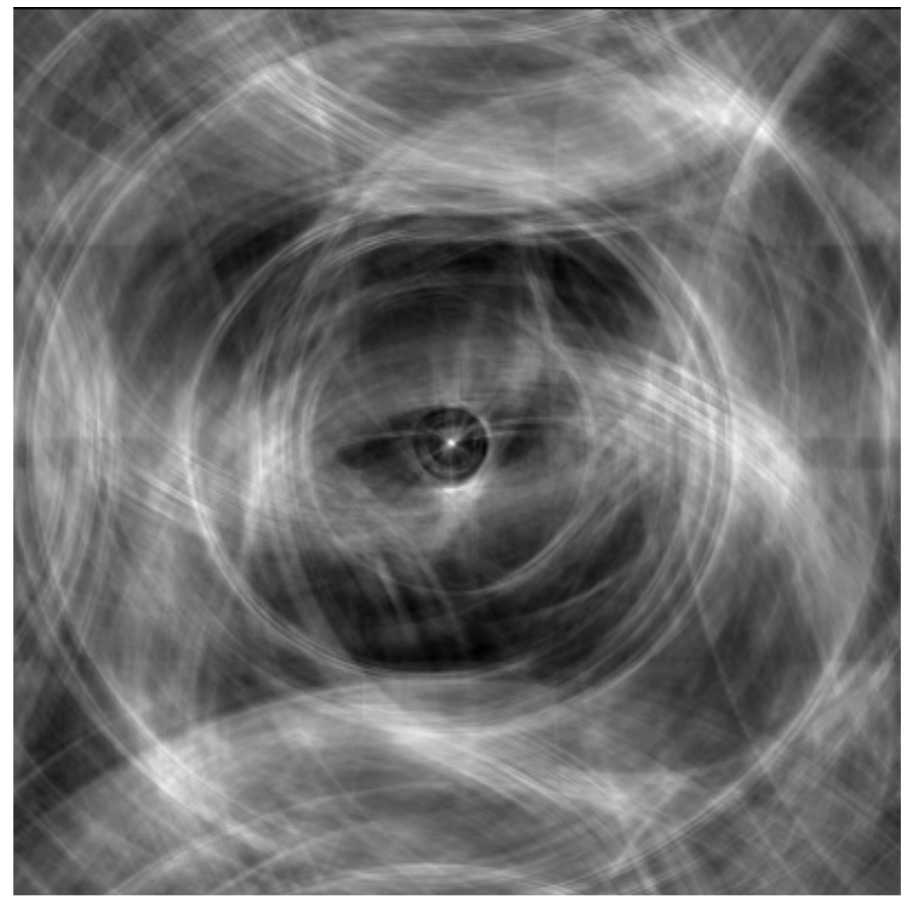

Fig. 4: Correlation plane output displaying a large number of spurious peaks of which only one is valid. 
radius of the alignment feature, the peak appears as a bright spot with a very small footprint or pedestal [13]. The correlation of mismatched circles may overlap with each other and create hotspots in the correlation plane.

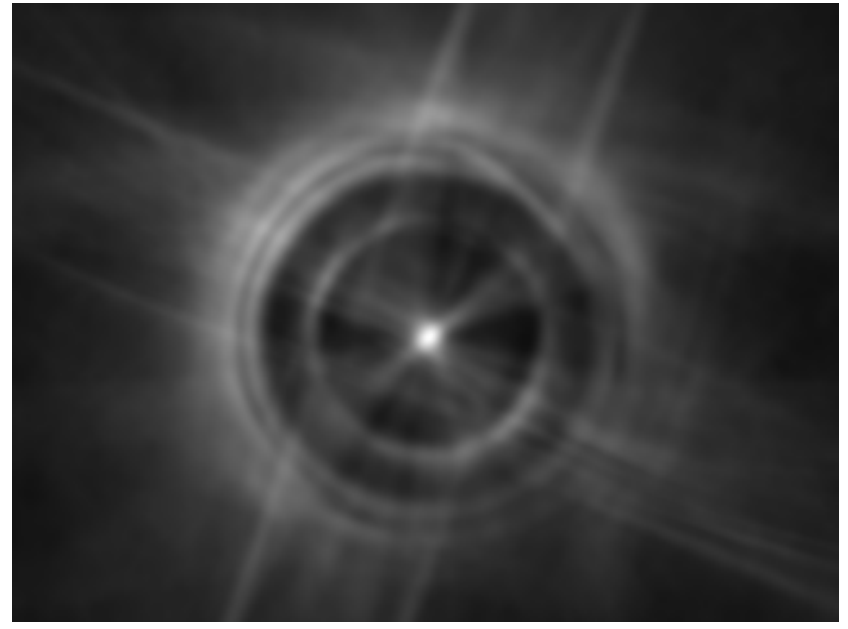

(a)

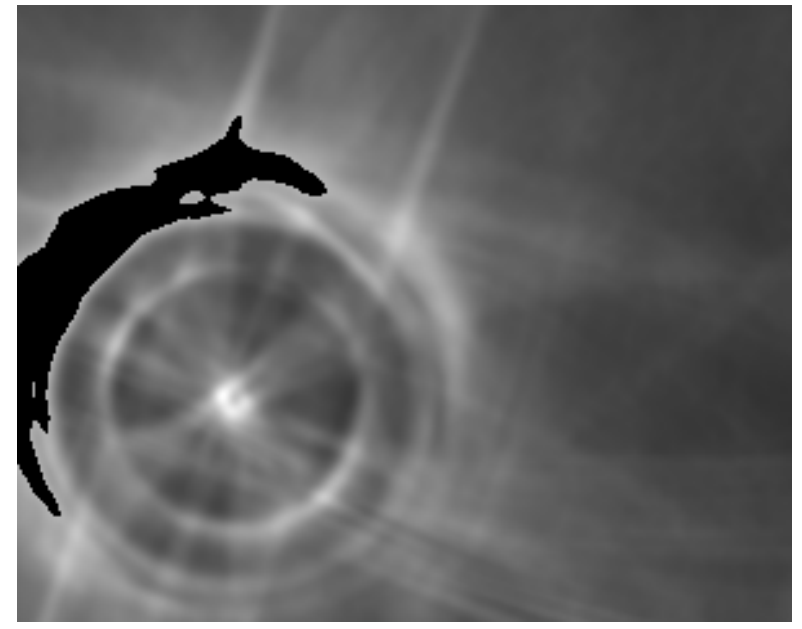

(b)

Fig. 5 (a) Segmented correlation plane. (b) Segmented correlation after passing through the peak filter.

To reduce the search area, it is noted that the hohlraum can occupy only certain location within the image, as such, the position of the peak must be bounded by certain region inside the image. Therefore, we segment this region from the correlation output for further processing as shown in Fig. 5(a). First, the segmented correlation output is passed through a peak filter. Its purpose is to identify regions in the correlation plane that most likely could contain spurious noise peaks. After thresholding at a $70 \%$ of the peak intensity, we calculate the spread of each candidate region. If the region spread is greater than a certain threshold, the pixels are set to zero as shown in Fig. 5(b). This step eliminates competing peaks appearing in those locations in the final location selection. After this step we perform a double peak detection and filter those out [13].

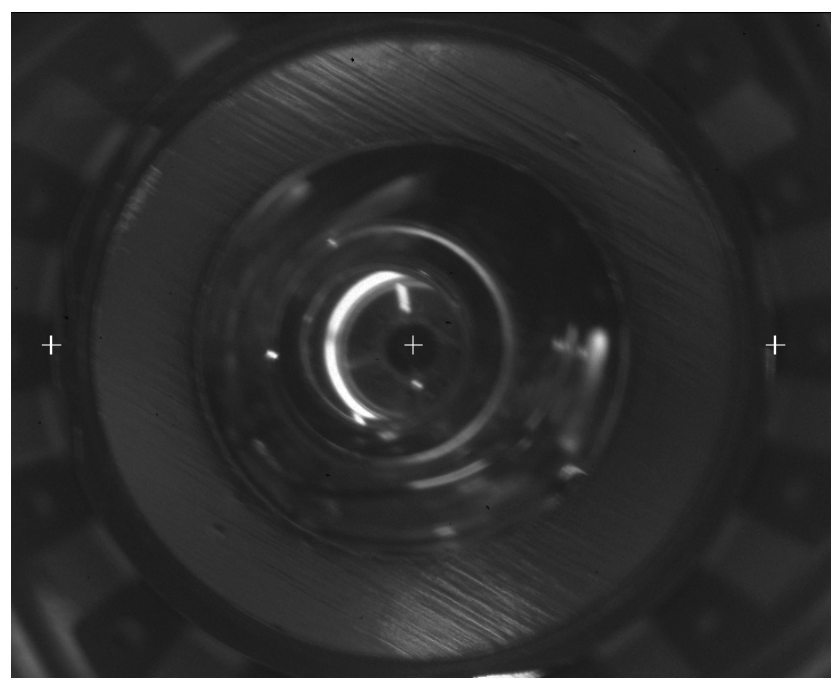

(a)

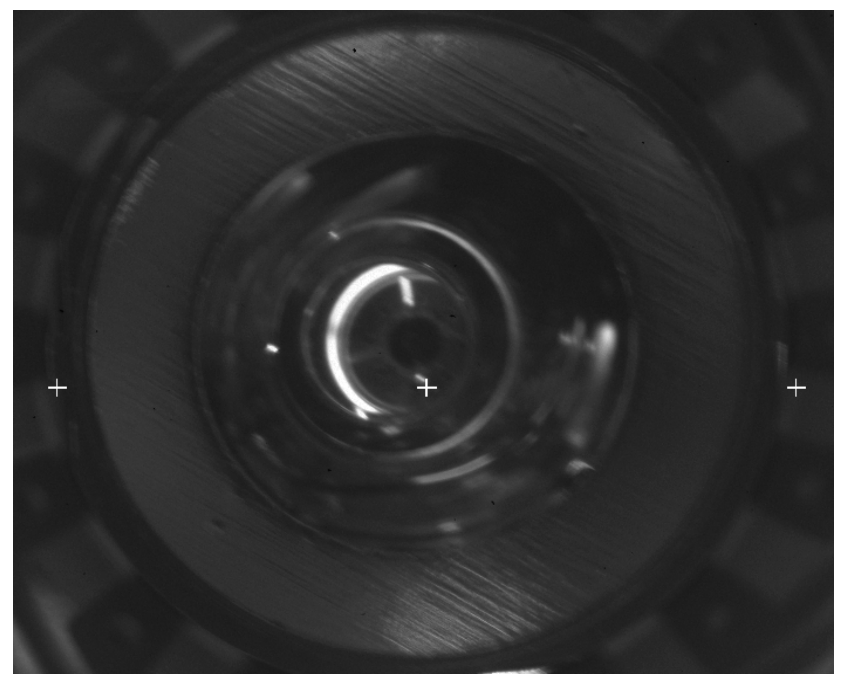

(b)

Fig. 6: Final position output of the hohlraum target with fiducial markers (a) with and (b) without peak filter 
This step ensures a reliable detection of the circular fiducial is found as shown in Fig. 6. One may be curious to know what happens when this step is eliminated. If we abandon the peak filter step as performed in Fig. 5(b), the maximum correlation peak chosen corresponds to partial match with the ring. As a result, although the two end points seem to be correctly placed as shown in Fig. 6(b), the center shows a clear bias downwards. In order to understand the spurious peak selection process, we examine the peak filter stage in more details.

After the peak filter, as shown in Fig. 7, a large region of spurious peaks is eliminated. The threshold is gradually raised until a single region is left. Keeping the peak on the left of Fig 7, the result of Fig. 6(a) is obtained.

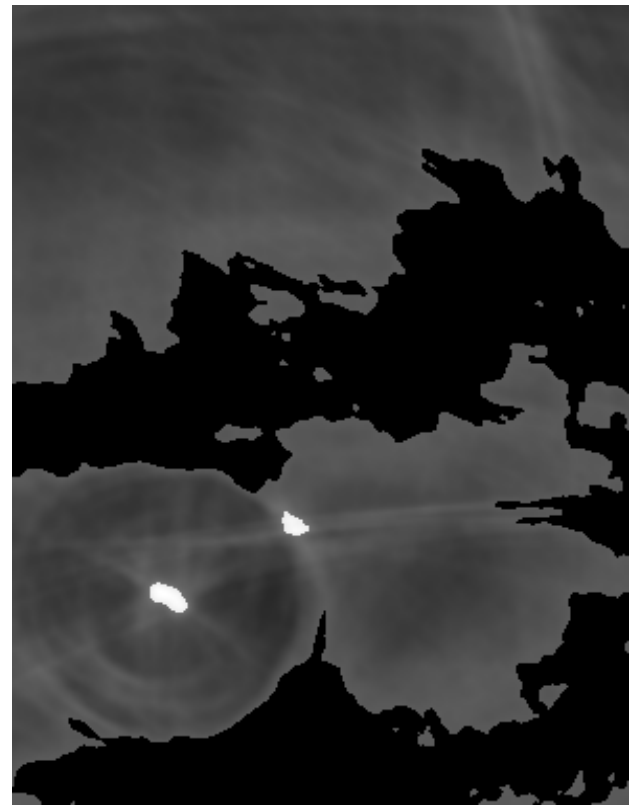

(a)

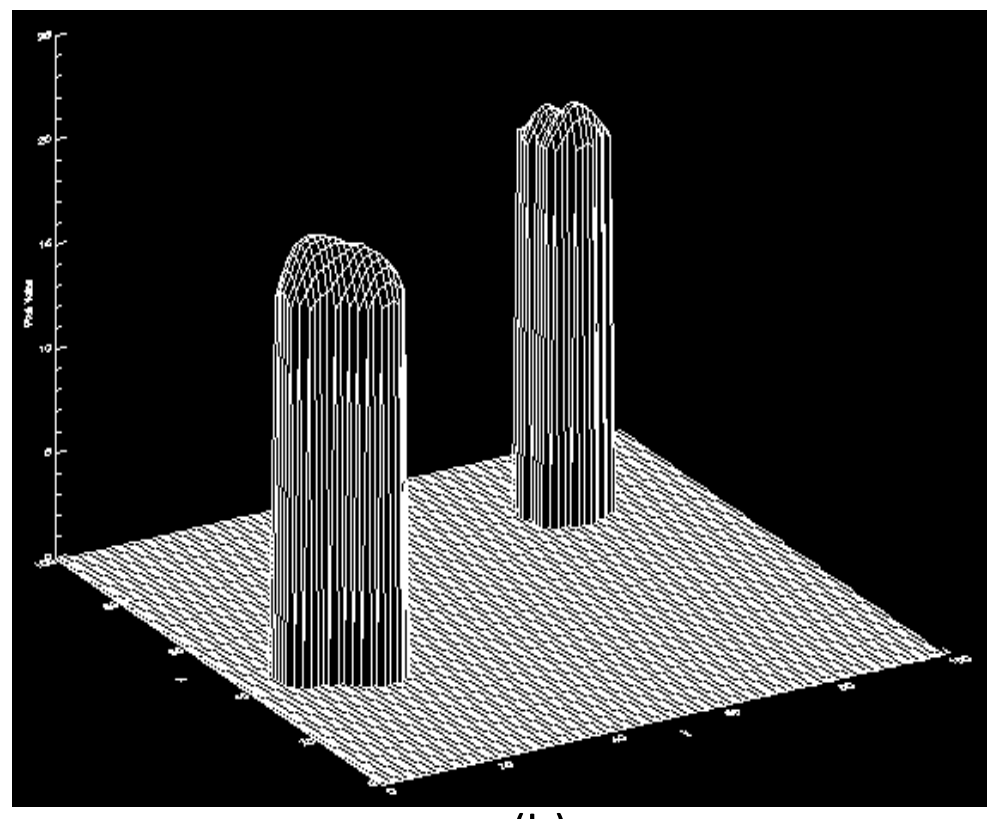

(b)

Fig. 7: Peak filtered correlation plane (a) showing dark region, only two competing peaks (b). These two regions are amplified 20 times to highlight their positions in correlation plane.

When peak filtering is not performed, the double peak detector finds two competing regions as shown in the left inset of Fig. 8. As the threshold is raised, the true peak which appears at the center of the segmented correlation plane starts to disappear. By the third iteration as depicted in the third inset of Fig. 8, the center has disappeared and two competing peaks are found, from which only one is chosen, which clearly results in a biased result as shown earlier in Fig. 6 (b).
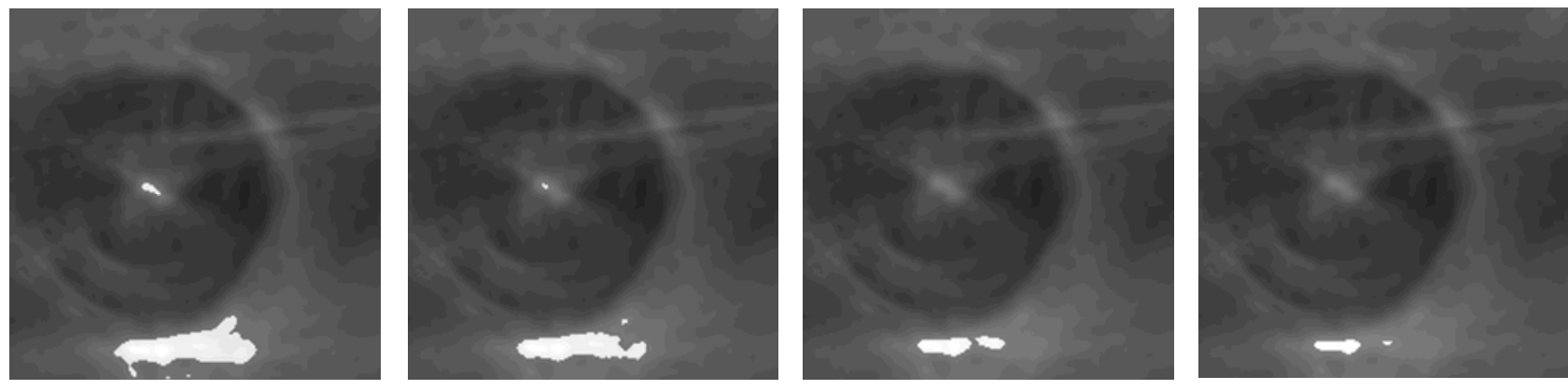

Fig. 8: Without the peak filter, the peak detector detects a peak on the periphery, unless these outside regions are removed. The real correlation peak is weaker and eliminated as shown on the third inset 


\section{CONCLUSIONS}

In this paper, an algorithm for detecting extremely faint fiducials in hohlraum images is described. It is demonstrated that matched filtering with additional spatial filtering of spurious peaks based on the broadness of correlation peak in the correlation domain leads to successful detection of extremely faint objects. Real-time operation is facilitated by executing these algorithms using externally supplied nominal radius and range as database parameters. This databasedriven real-time system, allows various sizes of hohlraum fiducials to be detected.

\section{ACKNOWLEDGEMENT}

This work performed under the auspices of the U.S. Department of Energy by Lawrence Livermore National Laboratory under Contract DE-AC52-07NA27344. AA acknowledges comments made by Steve Azevedo on this paper.

\section{REFERENCES}

1. E. I. Moses, "Overview of the National Ignition Facility", Fusion Science and Technology 54, pp. 361-366 (2008).

2. J. V. Candy, W. A. McClay, A. A. S. Awwal, and S. W. Ferguson, "Optimal position estimation for the automatic alignment of a high-energy laser," Journal of Optical Society of America A, Vol. 22, pp. 1348-1356, 2005.

3. R. A. Zacharias, N. R. Beer, E. S. Bliss, et al., "Alignment and wavefront control systems of the National Ignition Facility,” Optical Engineering 43, 2873-2884 (2004).

4. K. Wilhelmsen, A. Awwal, W. Ferguson, B Horowitz, V. Miller Kamm, C. Reynolds, "Automatic Alignment System For The National Ignition Facility", Proceedings of 2007 International Conference on Accelerator and Large Experimental Control Systems (ICALEPCS07), 486-490, Knoxville, Tennessee (2007). http://accelconf.web.cern.ch/accelconf/ica07/PAPERS/ROAA02.PDF

5. A. A. S. Awwal, Wilbert A. McClay, Walter S. Ferguson, James V. Candy, Thad Salmon, and Paul Wegner, "Detection and Tracking of the Back-Reflection of KDP Images in the presence or absence of a Phase mask," Applied Optics, Vol. 45, pp. 3038-3048, May 2006.

6. A. A. S. Awwal, "Automatic identification of templates in matched filtering," in Photonic Devices and Algorithms for Computing VI, edited by K. Iftekharuddin and A. A. S. Awwal,, Proc. of SPIE 5556, 102-109 (2004).

7. A. A. S. Awwal, "Multi-object feature detection and error correction for NIF automatic optical alignment" in Photonic Devices and Algorithms for Computing VIII, edited by K. Iftekharuddin and A. A. S. Awwal, Proc. SPIE 6310, 63100Q (2006).

8. A. A. S. Awwal, Kenneth L. Rice, Tarek M. Taha, "Fast implementation of matched-filter-based automatic alignment image processing", Optics \& Laser Technology 41, 193-197 (2009).

9. A. VanderLugt, "Signal Detection by Complex Spatial Filtering", IEEE Trans. Inf. Theory IT-10, 139-145 (1964).

10. H. L. Van Trees, Detection, Estimation, and Modulation Theory, Part I (Wiley, 2001).

11. A. A. S. Awwal, M. A. Karim, and S. R. Jahan, "Improved Correlation Discrimination Using an AmplitudeModulated Phase-Only Filter," Applied Optics 29, 233-236 (1990).

12. M. A. Karim and A. A. S. Awwal, Optical Computing: An Introduction, John Wiley, New York, NY, 1992.

13. A. A. S. Awwal, "What can we learn from the shape of a correlation peak for position estimation?," Applied Optics, Vol. 49, pp. B41-B50, April 1, 2010.

14. T. Wolf, B. Gutman, H. Weber, J. Ferre-Borrull, S. Bosch, and S. Vallmitjana, "Application of fuzzy-rule-based postprocessing to correlation methods in pattern recognition," Appl. Opt. 35, 6955-6963, (1996). 